\title{
Medialization Thyroplasty Using Nasal Septal Cartilage: Our Experience
}

\author{
Vinod T Kandakure ${ }^{1}$, Rucha M Joshi
}

\begin{abstract}
Aim: To demonstrate the functional voice outcomes following external medialization thyroplasty using autologous cartilage from the nasal septum.

Materials and methods: The study included four patients (three males and one female) who presented to the ENT outpatient department diagnosed with unilateral vocal fold paralysis. Medialization thyroplasty type I was performed for all the patients using autologous nasal septal cartilage with the patients under local anesthesia.

Results: All four patients showed significant improvement in the form of glottis closure on laryngoscopic examination. Complete glottis closure was achieved in three patients while one patient had a small posterior glottis gap. The maximum phonation duration improved significantly in three patients, i.e., average 13.33 seconds; while one patient with the small posterior glottis gap showed minor improvement, i.e., 7.1 seconds. However, the complaint of aspiration of liquids was relieved in all the four patients.

Conclusion: External medialization thyroplasty using an autologous nasal septal cartilage graft is considered safe and efficient phonosurgical procedure and provides proper positional adjustment of the paralyzed vocal fold with significant improvement in the subjective and objective functional voice outcomes.

Keywords: Laryngoscopy, Thyroplasty, Vocal fold paralysis.

International Journal of Phonosurgery \& Laryngology (2019): 10.5005/jp-journals-10023-1171
\end{abstract}

\section{INTRODUCTION}

Unilateral vocal cord paralysis is defined as paralysis of unilateral recurrent laryngeal nerve due to which the vocal cord on that side assumes a paramedian position with very less mobility or flickering movements. The causes of paralysis of a single vocal cord include thyroid surgery; carcinoma of cervical esophagus or thyroid; neck trauma; and cervical lymphadenopathy or mediastinal causes like bronchogenic carcinoma, aortic aneurysm, intrathoracic surgery, tuberculosis of cervical pleura, and idiopathic. ${ }^{1}$ It is most commonly caused due to neurological injury. The vocal cords cannot approximate fully due to the hypomobility of on cord, which causes impairment of phonation and difficulty in swallowing. This is called glottal incompetence. Most of the laryngeal functions are inhibited due to this incomplete closure of the glottis, including airway patency, respiration, and maintenance of the body core during physical activity. ${ }^{1}$ Due to inadequate compensatory action of the unparalyzed vocal fold, patients present with dysphonia and dysphagia including aspiration of liquids. Repeated episodes of aspiration can cause pneumonitis or other potentially lifethreatening complications. ${ }^{1}$ However, the important problem of the patient as well as the doctor is the speaking difficulty and vocal outcome. After the introduction of the technique of thyroplasty by Isshiki, laryngeal framework surgery has been used as a procedure of choice for the treatment of incomplete glottal closure.

Medialization thyroplasty is a standard treatment modality for cases of unilateral laryngeal palsy due to its durability and efficiency. The advantages of autologous or homologous grafts are biocompatibility, cost effectiveness, bioavailability, and lower chances of rejection of graft compared with alloplastic implants, which makes them the preferred choice for the procedure. The homologous material of choice for grafting is the cartilage as it is easily available, has low absorption rate, is biocompatible, and
1,2Department of ENT, Government Medical College, Latur, Maharashtra, India

Corresponding Author: Vinod T Kandakure, Department of ENT, Government Medical College, Latur, Maharashtra, India, Phone: +91 9130790522, e-mail: vinodkandakure@gmail.com

How to cite this article: Kandakure VT, Joshi RM. Medialization Thyroplasty Using Nasal Septal Cartilage: Our Experience. Int J Phonosurg Laryngol 2019;9(1):9-12.

Source of support: Nil

Conflict of interest: None

fibrosis is minimal due to which there is decreased shrinkage of tissue. ${ }^{1}$ The aim of the present study is to determine the functional vocal outcomes in patients who underwent external medialization thyroplasty using autologous nasal septal cartilage.

\section{Materials and Methods}

The study included four patients who attended the ENT outpatient department at our institute during the year 2016-2017 and who were diagnosed with unilateral vocal cord palsy. A written informed consent was taken from all the patients participating in the study about the surgical procedures, the expected results, and the possibility of complications. The patients were subjected to clinical examination, voice analysis, assessment of maximum phonation duration, and other laboratory investigations preoperatively. A detailed history was taken to look for possible etiology of the vocal cord palsy. Parameters that were noted with respect to voice were duration since change of voice, mode of onset, presence of hoarseness, breathiness, voice fatigue, pitch breaks, and level of loudness of voice. ${ }^{2}$ There were 3 males and 1 female included 
in the study. Three patients had left vocal cord palsy while one patient had right vocal cord palsy. The cause of vocal cord paralysis of three patients was unknown while one patient had a history of previous cardiac bypass surgery. All the patients had a history of vocal cord palsy since 1 year with no signs of improvement in voice quality with intermittent history of aspiration of liquids. All the patients were given voice therapy preoperatively. The patients were examined prior to surgery, at 1 week postsurgery and later after 1 month and 6 months. Before surgery, CT thorax, CT neck and chest, and MRI brain were done in all patients. Each patient underwent videolaryngoscopic examination, voice analysis, and aerodynamic assessment in the form of measurement of maximum phonation duration, preoperatively as well as postoperatively at every follow-up visit.

\section{Surgical Procedure}

\section{Procedure of Harvesting the Septal Cartilage}

Anesthesia: Infiltration was done in the following sites on both sides with $2-3 \mathrm{~mL}$ of anesthetic solution (2\% lignocaine with 1:100,000 adrenaline): intercartilaginous injection of the nasal dorsum from the region of the rhinion to the supratip region, at the base of the columella and the nasal tip, and was extended into the floor of the nose.

Surgical technique: A hemi-transfixion incision was taken to obtain the graft. The septal cartilage was harvested in one piece after carefully separating it from the maxillary crest inferiorly, the vomer posteroinferiorly, and the perpendicular plate of the ethmoid bone posterosuperiorly (Fig. 1). The cartilage was then remodeled by cutting it into multiple pieces of various sizes, largest being $4 \mathrm{~mm}$ $\times 13 \mathrm{~mm}$, and other smaller pieces for using them for augmentation as per requirement (Fig. 2).

\section{Medialization Thyroplasty Procedure}

Anesthesia: Infiltration anesthesia was given through injecting 2-3 mL of anesthetic solution (2\% lignocaine with 1:100,000 adrenaline) posterolaterally in the depth of the midpoint of the posterior border of the sternocleidomastoid muscle (at the level of the cricoid cartilage). Another $2-3 \mathrm{~mL}$ was infiltrated superiorly and inferiorly to the point of the primary site of infiltration. The same procedure was done on the other side also.

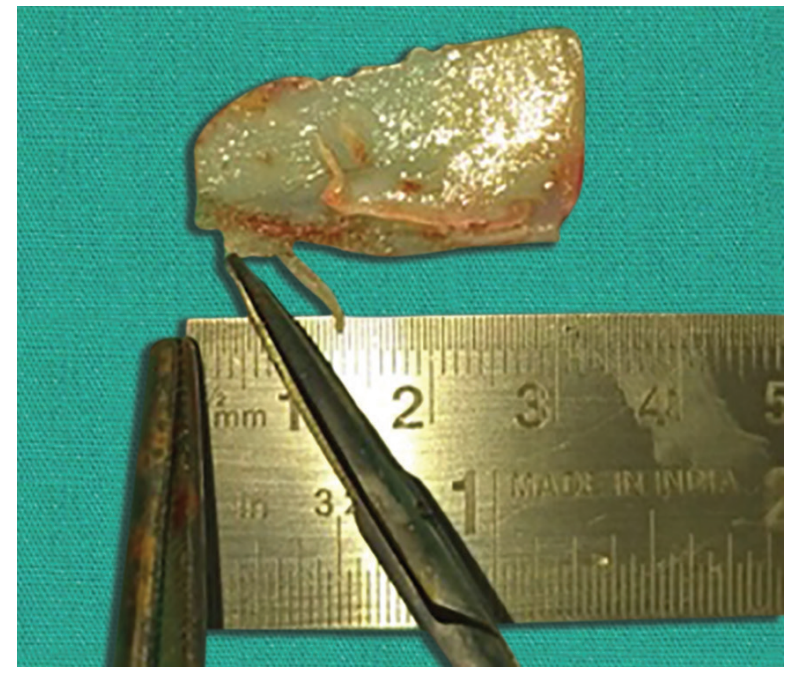

Fig. 1: Harvested nasal septal cartilage
Surgical technique: The technique given by Isshiki et al. was used for medialization of the vocal cord. One patient underwent thyroplasty on the right side, while in three patients, it was done on the left side. A horizontal neck incision was taken at the level in the midline of the thyroid cartilage. Blunt dissection was continued after dissecting the platysma first, then the strap muscles were retracted and the thyroid lamina was exposed. A perichondrial rectangle (window) was made at the level of the vocal folds ( $6 \mathrm{~mm}$ from the midline) and it measured $5 \mathrm{~mm}$ (height) $\times 12 \mathrm{~mm}$ (length). Later, the cartilage window was subsequently created. Using a Freer's elevator, the inner perichondrium was circumferentially elevated in a careful way to avoid injuring it. The cartilage graft of size $4 \mathrm{~mm} \times 13 \mathrm{~mm}$ was then carefully tucked into the window superficial to the inner perichondrium. To assess the best possible implant position intraoperatively, a perceptual evaluation of the patient's voices was done and further layers of the graft were inserted if needed to achieve the best voice quality the patient can attain. Intraoperative perceptual evaluation was carried out by asking the patient to count from 1 to 10 and recite a standard poetry that was memorized by all the patients previously. The patient's voice quality was judged by consensus agreement between the surgeon and a phoniatrician, who attended the surgical procedures. All the patients received wound drainage for approximately 2 days and they were observed in the hospital after surgery for an average of 3 days. The patients were evaluated at 1 week after surgery and then at 1 month and 6 months follow-up.

\section{Results}

The study included four patients ( 3 males and 1 female) who were found to have unilateral vocal cord palsy. Type I medialization thyroplasty was performed for all the patients using autologous nasal septal cartilage with the patients under local anesthesia. Preoperatively, the laryngoscopic examination revealed glottic gap in all patients (Fig. 3A). The maximum phonation durations of the patients were 3.2 seconds, 4.1 seconds, 2.6 seconds, and 3.6 seconds, respectively (average 3.375 seconds). These patients were operated for medialization thyroplasty using autologous nasal septal cartilage on left side in 3 patients (having left vocal cord palsy) and on right side in one patient (having right vocal cord palsy). On follow-up 1 week, 1 month and 6 months postoperatively, all

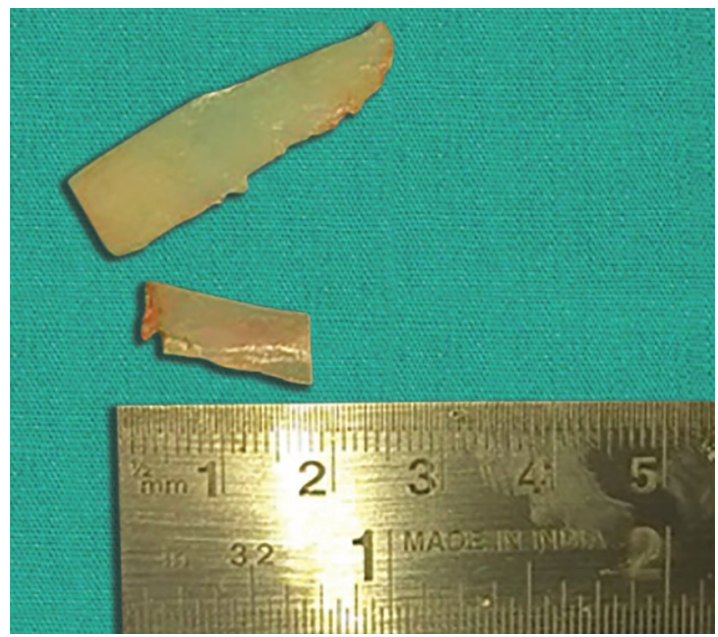

Fig. 2: Remodeled nasal septal cartilage, multiple pieces of various sizes, largest of $4 \mathrm{~mm} \times 13 \mathrm{~mm}$ 

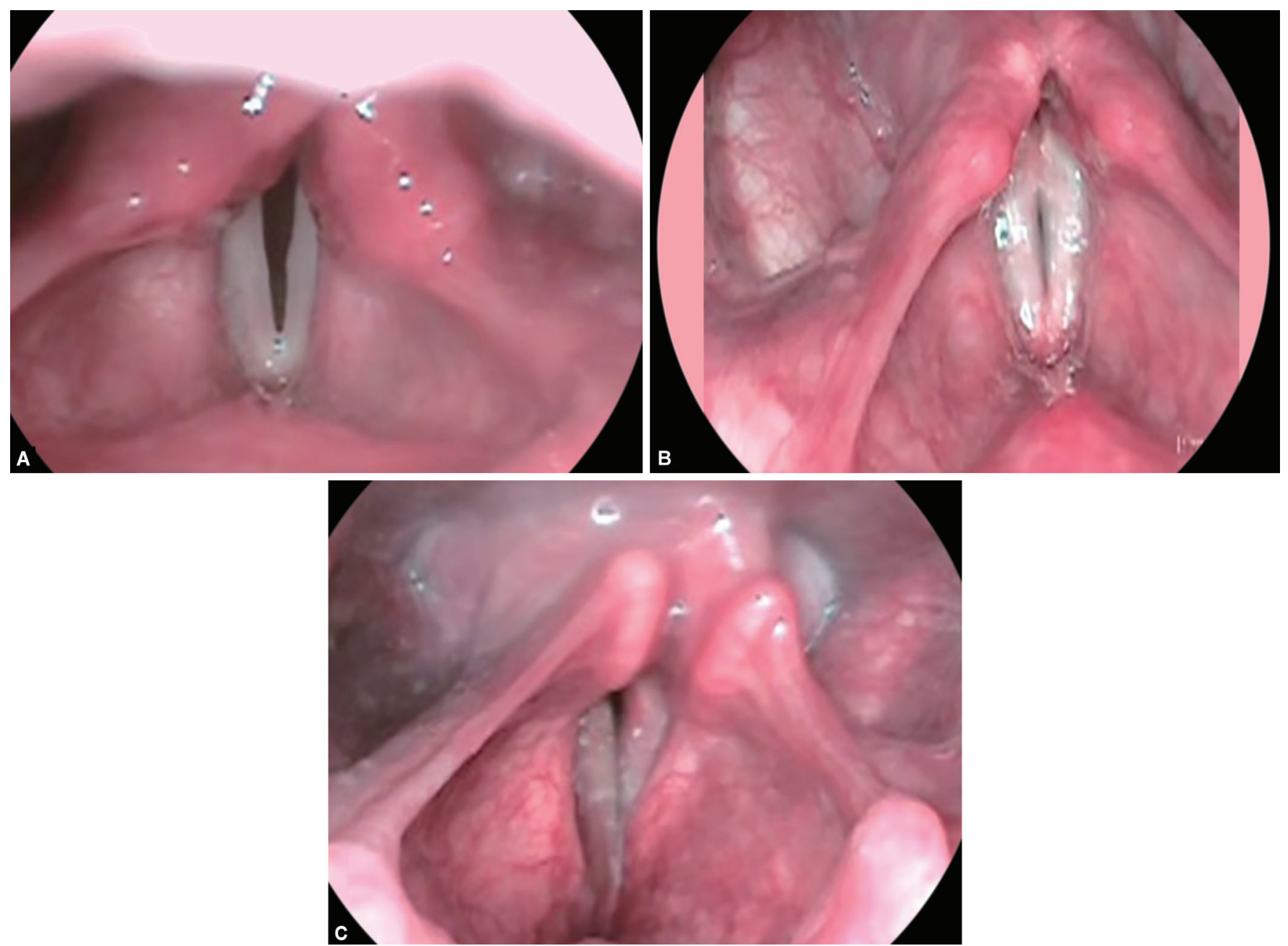

Figs 3A to C: (A) Preoperative glottal gap present in all four patients; (B) Postoperative complete glottal closure observed in three patients; (C) Postoperative posterior glottal gap in one patient

four patients showed significant improvement in the form of glottis closure on laryngoscopic examination. Complete glottis closure was achieved in three patients (Fig. 3B) while one patient had a small posterior glottis gap (Fig. 3C). Significant improvement was noted in voice quality of three patients while one patient having small posterior glottis gap had slightly husky voice. The maximum phonation duration improved significantly in three patients, i.e., 13.6 seconds, 12.9 seconds, and 13.5 seconds, respectively (average 13.33 seconds), while one patient with the small posterior glottis gap showed minor improvement, i.e., from 3.6 seconds to 7.1 seconds (Table 1). However, the complaint of aspiration of liquids was relieved in all the four patients.

\section{Discussion}

Voice disorders due to vocal cord palsy are very common. Majority of the patients live with this condition as they are ignorant about the treatment. Laryngeal framework surgeries are one of the foremost advances of recent times. It allows changes in the glottis aperture, tension in the cords, and length of the cords without any direct intervention on the cords. Thus, it avoids manipulating the dynamic vibratory structure of the cords. ${ }^{2}$

The current treatment for one-sided laryngeal palsy includes medialization thyroplasty with or without arytenoid adduction (AA), injection laryngoplasty, laryngeal reinnervation, adduction arytenopexy, and cricothyroid subluxation. A 6-12month observation period is generally suggested before permanent medialization thyroplasty. During the 6-12-month waiting period, trial of vocal cord injection with autologous fat or hyaluronic acid is a valid option with favorable but short-term results; however, this treatment option is less suitable for patients with large posterior glottal gaps, as its long-lasting benefit is controversial. ${ }^{1}$ Furthermore, laryngeal reinnervation has been reported to have less favorable results in the elderly and those with atrophy of the laryngeal muscles and to have a longer interval between injury and reinnervation. Therefore, medialization thyroplasty is a standard treatment when long-lasting improvement is required and for achieving adequate glottal closure and particularly for wide glottal gaps. Moreover, there are some advantages of external medialization thyroplasty over other techniques such as the former can be conducted under local anesthesia, and the implant is placed lateral to the inner perichondrium of the thyroid cartilage lamina, so that the structural integrity of the vocal cord can be preserved. Also, it has been reported that the endoscopic augmentation techniques induce vocal fold stiffness with loss of the mucosal waves.

Isshiki type I thyroplasty with nonresorbable biomaterials, such as silicone, Gore-Tex, hydroxyapatite, titanium, and expanded 
Table 1: Summary of the results

\begin{tabular}{|c|c|c|c|c|c|c|}
\hline Patient & Age & Sex & $\begin{array}{l}\text { Etiology of vocal } \\
\text { cord paralysis }\end{array}$ & $\begin{array}{l}\text { Preoperative } \\
\text { maximum } \\
\text { phonation } \\
\text { duration (seconds) }\end{array}$ & $\begin{array}{l}\text { Postoperative } \\
\text { maximum } \\
\text { phonation } \\
\text { duration (seconds) }\end{array}$ & Glottis closure \\
\hline 1 & 55 years & Male & $\begin{array}{l}\text { Postcardiac } \\
\text { surgery }\end{array}$ & 3.2 & 13.6 & Adequate \\
\hline 2 & 42 years & Female & $\begin{array}{l}\text { Postthyroid } \\
\text { surgery }\end{array}$ & 4.1 & 12.9 & Adequate \\
\hline 3 & 58 years & Male & Idiopathic & 2.6 & 13.5 & Adequate \\
\hline 4 & 47 years & Male & Idiopathic & 3.6 & 7.1 & Inadequate \\
\hline
\end{tabular}

polytetrafluoroethylene, has also been tried in the past few years. ${ }^{3}$ Although these materials are effective for vocal improvement, there are associated disadvantages such as graft rejection and some potential complications. Therefore, highly biocompatible autologous or homologous grafts are necessary, which are costeffective, easily available, and associated with lesser chances of rejection compared with alloplastic implants. Autologous grafts are freely available, have no risk of tissue rejection, and are highly suitable for medialization thyroplasty. Amongst them, the cartilage is a preferred graft material for the same, because of its easy availability, low absorption rate, high biocompatibility, and minimal tissue reaction or fibrosis which causes minimal shrinkage. ${ }^{2}$

The appropriate timing for external medialization thyroplasty is controversial because of the relative difficulty in determining the type of recurrent laryngeal nerve injury. Therefore, in such situations, it is recommended to assess the nerve conduction by performing electromyography to exclude complete nerve degeneration and loss. ${ }^{4}$ Partial recovery can be expected in some cases in which the nerve is not completely damaged. Functional voice therapy can successfully restore better voice quality in many patients with unilateral vocal cord paralysis and especially if the glottal gap is insignificant. Medialization thyroplasty is, thus, mainly indicated for patients with a large glottal gap and after voice therapy has failed to achieve sufficient closure of the glottis.

Medialization thyroplasty was carried out using autologous cartilage from the nasal septum as an implant. Because of its better tissue tolerability, an autologous cartilage implant is less likely to be rejected or extruded. It can be easily cut and shaped with the scalpel to be easily adjusted into the thyroid cartilage window. The cartilage implant does not need any external fixation as it fits nicely and can be easily introduced under the edges of the thyroid lamina window. ${ }^{5}$ Multiple layers of the cartilage can be inserted for augmentation according to the degree of medialization required for the patient as guided by the intraoperative voice quality assessment. Except for a small endolaryngeal hematoma that is frequently observed after surgery and as this resolves spontaneously with time, compared to other studies, there were no major complications in our patients as well. ${ }^{6}$

Arytenoid adduction is sometimes done by surgeons along with medialization thyroplasty to improve the posterior glottic closure. However, many studies have suggested the importance of AA in improving the objective or subjective results of thyroplasty surgical procedures. ${ }^{7}$ Moreover, a recent study has reported no significant difference in the postoperative closure of the glottis for the patients who underwent only thyroplasty and those who underwent thyroplasty with $A A^{8}{ }^{8}$ In this present study, there was no need for supplementary AA as all of our patients achieved complete glottal closure postoperatively and they were satisfied with their new voices. This indicates the minor role of the respiratory glottis in affecting the voice quality of patients following thyroplasty procedures.

\section{Conclusion}

Medialization thyroplasty using an autologous graft obtained from the nasal septal cartilage is considered a secure, harmless, and competent procedure of phonosurgery. It can be used for the correction of glottal insufficiency in cases of unilateral vocal cord palsy with an extra advantage of not harming the structural integrity of the vocal cords. This technique provides proper position to the paralyzed vocal cord in view of approximation with the normal vocal cord and thus significantly improves the subjective and objective functional vocal outcomes as well as provides satisfactory relief in view of aspiration of liquids.

\section{References}

1. Tsai M-S, Yang M-Y, Chang G-H, et al. Autologous thyroid cartilage graft implantation in medialization laryngoplasty: a modified approach for treating unilateral vocal fold paralysis. Sci Rep 2017;7(1):4790. DOI: 10.1038/s41598-017-05024-6.

2. Raj A, Girhotra M, Meher R. Medialisation laryngoplasty - a study of 15 cases. Indian J Otolaryngol Head Neck Surg 2004;56(4):283-288. DOI: 10.1007/BF02974388.

3. Elnashar I, El-Anwar M, Amer H, et al. Voice outcome after goretex medialization thyroplasty. Int Arch Otorhinolaryngol 2015;19: 248-254. DOI: 10.1055/s-0034-1397339.

4. Varvares MA, Brandsted RM. Medialisation thyroplasty using the montgomery thyroplasty system. Springer, Berlin, Heidelberg; 2006, ch. 9; 9.2. pp. 153-154.

5. Mesallam TA, Khalil YA, Malki KH, et al. Medialisation Thyroplasty using autologous nasal septal cartilage for treating unilateral vocal fold paralysis. Clin Exp Otorhinolaryngol 2011;4(3):142-148. DOI: 10.3342/ceo.2011.4.3.142.

6. Behrman A. Evidence-based treatment of paralytic dysphonia: making sense of outcomes and efficacy data. Otolaryngol Clin North Am 2004;37(1):75-104, vi 10.1016/S0030-6665(03)00169-5.

7. Li AJ, Johns MM, Jackson-Menaldi C, et al. Glottic closure patterns: type I thyroplasty versus type I thyroplasty with arytenoid adduction. J Voice 2011;25(3):259-264. DOI: 10.1016/j.jvoice.2009.11.001.

8. Schneider B, Denk DM, Bigenzahn W. Functional results after external vocal fold medialization thyroplasty with the titanium vocal fold medialization implant. Laryngoscope 2003;113(4):628-634. DOI: 10.1097/00005537-200304000-00008. 